\title{
The Application of Matrix in Control Theory
}

\author{
Yuanyuan Zhang \\ College of Science, China Three Gorges University, Yichang, China
}

Email address:

mathzhyy@163.com

\section{To cite this article:}

Yuanyuan Zhang. The Application of Matrix in Control Theory. Applied and Computational Mathematics. Vol. 5, No. 3, 2016 , pp. 165-168. doi: $10.11648 /$ j.acm.20160503.21

Received: June 21, 2016; Accepted: July 21, 2016; Published: July 25, 2016

\begin{abstract}
This paper presents a new way to justify the controllability of linear ordinary systems. This way is based on the maximum geometric multiplicity of eigenvalues for the coefficient matrix of the linear ordinary equation. This method is equivalent to other discrimination laws for controllability.
\end{abstract}

Keywords: Controllability, Ordinary Differential Equation, Geometric Multiplicity, Eigenvalues

\section{Introduction}

The controllability problems of ordinary differential equations have been a great interest for decades due to their practical applications [1-5]. There are a lot of literatures on this issue ( see for instance [6-15]). The current paper presents a new way to justify the controllability of linear ordinary systems. This way is based on the maximum geometric multiplicity of eigenvalues for the coefficient matrix of the linear ordinary equation.

The rest of the paper is organized as follows: Section 2 presents a necessary and sufficient condition for justifying the controllability of the linear ordinary differential system. Section 3 gives several examples to show the efficiency of the new method. Section 4 is the conclusion of this paper.

\section{Main Results}

Let $A$ and $B$ be two matrix in $R^{n \times n}$ and $R^{n \times m}$ respectively, where $n, m \in N$. Consider the following controlled ordinary differential equation:

$$
\left\{\begin{array}{c}
y^{\prime}(t)+A y(t)=B u(t) \\
y(0)=y_{0}
\end{array}\right.
$$

Here $y_{0} \in R^{n} \backslash\{0\}$ and $u$ is a control function in the following admissible set:

$$
\mu_{M}=\left\{v:[0,+\infty) \rightarrow R^{m},\|v(t)\|_{R^{M}} \leq M\right\},
$$

where $M$ is a positive constant. Throughout this paper, we use
$(A, B)$ to denote the linear differential system given by equation (1). The main result of the paper is as follows:

Theorem The linear differential system $(A, B)$ is controllable if and only if the number of columns of the matrix $B$ is no less than the maximum geometric multiplicity of eigenvalues for the matrix $A$.

Proof. Suppose the system $(A, B)$ is controllable, and $\lambda_{1}, \lambda_{2}, \cdots \lambda_{q}$ are the eigenvalues of the matrix $A$ with the geometric multiplicity $m_{1}, m_{2}, \cdots m_{q}$ and algebraic multiplicity $n_{1}, n_{2}, \cdots n_{q}$. Then there exists an invertible matrix $P$ such that

$$
\tilde{A} \triangleq P^{-1} A P=\left(\begin{array}{llll}
J_{1} & & & \\
& J_{2} & & \\
& & \ddots & \\
& & & J_{q}
\end{array}\right),
$$

where

$$
J_{k}=\left(\begin{array}{cccc}
L_{1}^{k} & & & \\
& L_{2}^{k} & & \\
& & \ddots & \\
& & & L_{m}^{k}
\end{array}\right), \quad(k=1,2, \cdots, q) .
$$

In equation (4) $L_{j}^{k}$ represents the Jordan's block corresponding to $\lambda_{k},(k=1,2, \cdots, q)$.

Suppose the order of the Jordan block $L_{j}^{k}$ is $l_{j}^{k}$, and

$$
l_{1}^{k}+l_{2}^{k}+\cdots l_{m_{k}}^{k}=n_{k},(k=1,2, \cdots, q) \text {. }
$$

Let $\tilde{B} \triangleq P^{-1} B$. We use $\left(P_{1}\right)$ to represent the system 
$(A, B)$ is controllable and $\left(P_{2}\right)$ to represent the system $(\widetilde{\mathrm{A}}, \widetilde{B})$ is controllable, then the problems $\left(P_{1}\right)$ and $\left(P_{2}\right)$ are equivalent.

Suppose $(\widetilde{A}, \widetilde{B})$ is controllable, then for any eigenvalues $\lambda$, take $\lambda_{1}$ for example, we have

$$
\left(\lambda_{1}-\tilde{A}, \tilde{B}\right)=\left(\begin{array}{ccc}
\lambda_{1} I-J_{1} & 0 & \tilde{B}_{1} \\
0 & \lambda_{1} I-C & \tilde{B}_{2}
\end{array}\right)
$$

where

$$
\operatorname{rank}\left(\lambda_{1} I-\tilde{A}_{1}, \quad 0, \quad \tilde{B}_{1}\right)=n_{1} .
$$

It is obvious that the number of nonzero rows for equation (6) is $m_{1}$, where $m_{1} \leq n_{1}$. Then from equation (6) we can see that the number of linearly independent rows of the matrix $\tilde{B}_{1}$ must be no less than $m_{1}$. So the number of columns for equation $B$ must be no less than $m_{1}$. Because $\lambda_{1}$ is any eigenvalue for the matrix $A$, so the necessity of the theorem has been proved.

On the other hand, if the number of columns for equation $B$ is no less than $m_{k},(k=1,2, \cdots, q)$, then for any eigenvalues $\lambda_{k}$, we have

$$
\operatorname{rank}\left(\lambda_{k} I-\tilde{A}, \quad \tilde{B}_{1}\right)=n_{k}
$$

So

$$
\operatorname{rank}(\lambda I-\tilde{A}, \quad \tilde{B})=n_{1}+n_{2}+\cdots+n_{k}
$$

From equation (9) we can obtain that all the rows of the matrix $\left(\widetilde{B}, \tilde{A} \tilde{B}, \cdots \tilde{A}^{n-1} \tilde{B}\right)$ are linearly independent, which completes the proof of the sufficiency of the theorem.

\section{Applications}

In this section we provide several examples to show the efficiency of the above-mentioned method.

Example 1. Let

$A=\left(\begin{array}{cccccc}4 & -5 & 2 & 0 & 0 & 0 \\ 6 & -7 & 3 & 0 & 0 & 0 \\ 6 & -9 & 4 & 0 & 0 & 0 \\ 0 & 0 & 0 & 0 & 1 & 0 \\ 0 & 0 & 0 & -4 & 4 & 0 \\ 0 & 0 & 0 & -2 & 1 & 2\end{array}\right), B=\left(\begin{array}{ccc}1 & 0 & 1 \\ 0 & 1 & 4 \\ 3 & 5 & 2 \\ 2 & 1 & -1 \\ 4 & 3 & 2 \\ -2 & 6 & 3\end{array}\right)$.

We aim to proof that system (10) is controllable.

Proof. Let

$$
A_{1}=\left(\begin{array}{lll}
4 & -5 & 2 \\
6 & -7 & 3 \\
6 & -9 & 4
\end{array}\right), A_{2}=\left(\begin{array}{ccc}
0 & 1 & 0 \\
-4 & 4 & 0 \\
-2 & 1 & 2
\end{array}\right),
$$

then the system (10) can be simplified as

$$
A=\left(\begin{array}{cc}
A_{1} & 0 \\
0 & A_{2}
\end{array}\right) \text {. }
$$

It is easy to obtain

$$
\begin{aligned}
\lambda E-A_{1} & =\left(\begin{array}{ccc}
1 & 0 & 0 \\
0 & 1 & 0 \\
0 & 0 & \lambda^{2}(\lambda-1)
\end{array}\right) \\
\lambda E-A_{2} & =\left(\begin{array}{ccc}
1 & 0 & 0 \\
0 & \lambda-2 & 0 \\
0 & 0 & (\lambda-2)^{2}
\end{array}\right) .
\end{aligned}
$$

So the Jordan's normal form of matrix $A$ is as follows

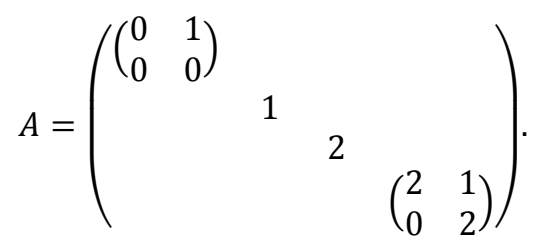

From equation (15), it is obvious that the maximum geometric multiplicity of the eigenvalues of the matrix $A$ is 2, and the number of the non-zero columns of the matrix $B$ is 3 . According to the given method in section 2, system (10) is controllable.

Example 2. Let

$$
A=\left(\begin{array}{cccc}
a_{1} & a_{2} & \cdots & a_{n} \\
0 & a_{1} & \cdots & a_{n-1} \\
\vdots & \vdots & \vdots & \vdots \\
0 & 0 & \cdots & a_{1}
\end{array}\right)_{n \times n}, B=\left(\begin{array}{cc}
1 & 0 \\
0 & 1 \\
\vdots & \vdots \\
0 & 0
\end{array}\right)_{n \times 2} .
$$

Our aim is to proof that system (16) is controllable.

Proof. For

$$
f(\lambda)=|\lambda E-A|=\left(\lambda-a_{1}\right)^{n}
$$

then the minimum polynomials of the matrix $A$ is

$$
m_{A}=\left(\lambda-a_{1}\right)^{k},(1 \leq k \leq n) .
$$

Let

$$
g(\lambda)=\left(\lambda-a_{1}\right)^{n-1}
$$

then

$$
g(A)=\left(A-a_{1} E\right)^{n-1}=\left(\begin{array}{cccc}
0 & \cdots & \cdots & a_{2}^{n-1} \\
& \ddots & & \vdots \\
& & \ddots & \vdots \\
& & & 0
\end{array}\right) \neq 0 .
$$

So

$$
m_{A}=\left(\lambda-a_{1}\right)^{n} .
$$

From equation (21), it is easy to obtain

$$
\lambda E-A \simeq\left(\begin{array}{cccc}
1 & & & \\
& \ddots & & \\
& & 1 & \left(\lambda-a_{1}\right)^{n}
\end{array}\right)_{n \times n} .
$$

So the elementary divisors of the matrix $A$ is

$$
\left(\lambda-a_{1}\right)^{n} .
$$

Then the Jordan's normal form of the matrix $A$ is as follows 


$$
\left(\begin{array}{cccc}
a_{1} & 1 & & \\
& \ddots & \ddots & \\
& & \ddots & 1 \\
& & & a_{1}
\end{array}\right) .
$$

From equation (24), it is easy to obtain the maximum geometric multiplicity of the eigenvalues of the matrix $A$ is 1, and the number of the non-zero columns of the matrix $B$ is 2 . So according to the given method in section 2 , system (16) is controllable.

Example 3. Let

$$
A=\left(\begin{array}{ccc}
-5 & 1 & 4 \\
-12 & 3 & 8 \\
-6 & 1 & 5
\end{array}\right), \quad B=\left(\begin{array}{l}
1 \\
0 \\
0
\end{array}\right)
$$

Our aim is to proof that system (25) is non-controllable.

Proof. For

$$
|\lambda E-A|=(\lambda-1)^{3},
$$

and the minimum polynomials of the matrix $A$ is

$$
m_{A}=(\lambda-1)^{2}
$$

then the invariant factors of the matrix $A$ is

$$
d_{1}(\lambda)=1, d_{2}(\lambda)=\lambda-1, d_{3}(\lambda)=(\lambda-1)^{2} .
$$

So the Jordan's normal form of matrix $A$ is as follows

$$
A=\left(\begin{array}{lll}
1 & 0 & 0 \\
0 & 1 & 1 \\
0 & 0 & 1
\end{array}\right)
$$

From equation (29), it is obvious that the maximum geometric multiplicity of the eigenvalue of the matrix $A$ is 2 , and the number of the non-zero columns of the matrix $B$ is 1 . According to the given method in section 2, system (25) is non-controllable.

Example 4. Let

$$
A=\left(\begin{array}{cccccc}
a & 1 & & & & \\
0 & a & & & & \\
& & b & 1 & & \\
& & 0 & b & & \\
& & & & c & 1 \\
& & & & 0 & c
\end{array}\right), B=\left(\begin{array}{l}
1 \\
0 \\
0 \\
0 \\
0 \\
0
\end{array}\right) .
$$

Proof. For system (30), there are different cases:

Case 1. When $a=b=c$, the maximum geometric multiplicity of the eigenvalue of the matrix $A$ is 3 , and the number of the non-zero columns of the matrix $B$ is 1 . According to the given method in section 2, system (30) is non-controllable.

Case 2. When $a=b \neq c$, the maximum geometric multiplicity of the eigenvalue of the matrix $A$ is 2 and the number of the non-zero columns of the matrix $B$ is 1 . According to the given method in section 2, system (30) is non-controllable.

Case 3. When $a \neq b \neq c$, the maximum geometric multiplicity of the eigenvalue of the matrix $A$ is 1 and the number of the non-zero columns of the matrix $B$ is 1 .
According to the given method in section 2, system (30) is controllable.

In (30), if the number of the non-zero columns of the matrix $B$ is 2 , for example,

$$
B=\left(\begin{array}{ll}
1 & 0 \\
0 & 1 \\
0 & 0 \\
0 & 0 \\
0 & 0 \\
0 & 0
\end{array}\right)
$$

then in case 1 the system $(A, B)$ is non-controllable; in case 2 and case 3 the system $(A, B)$ is controllable.

Still in (30), if the number of the non-zero columns of the matrix $B$ is 3 , for example,

$$
B=\left(\begin{array}{lll}
1 & 0 & 0 \\
0 & 1 & 0 \\
0 & 0 & 1 \\
0 & 0 & 0 \\
0 & 0 & 0 \\
0 & 0 & 0
\end{array}\right)
$$

then the number of non-zero columns of the matrix $B$ is 3 . According to the given method in section 2, whether $a, b, c$ are equal or not, the system $(A, B)$ is controllable.

In fact, as long as the number of non-zero columns of the matrix $B$ is no less than 3 , the system $(A, B)$ is always controllable.

\section{Conclusion}

This paper presents a new necessary and sufficient condition for justifying the controllability of the linear ordinary differential system. This way is based on the maximum geometric multiplicity of eigenvalues for the coefficient matrix of the linear ordinary equation. This method is equivalent to other discrimination laws for controllability. Section 2 presents a necessary and sufficient condition for justifying the controllability of the linear ordinary differential system. Section 3 gives several examples to show the efficiency of the new method. Section 4 is the conclusion of this paper. This method can also be used in other linear ordinary differential systems.

\section{References}

[1] V. Barbu, Analysis and Control of Nonlinear Infinite Dimensional Systems, Academic Press, Boston, 1993, pp. 74-98.

[2] L. C. Evans, An Introduction to Mathematical Optimal Control Theory, Lecture Notes, Department of Mathematics, University of California, Berkeley, 2008, pp. 92-101.

[3] H. O. Fattorini, Infinite Dimensional Linear Control Systems, the Time Optimal and Norm Optimal Control Problems, North-Holland Mathematics Studies 201, Elsevier, 2005, pp. 48-67. 
[4] K. Ito and K. Kunisch, "Semismooth newton methods for time-optimal control for a class of ODES", SIAM J. Control Optim., vol. 48, 2010, pp. 3997-4013.

[5] C. Y. Kaya and J. L. Noakes, "Computations and time-optimal controls", Optimal Control Applications and Methods, vol.17, 1996, pp.171-185.

[6] C. Y. Kaya and J. L. Noakes, "Computational methods for time-optimal switching controls", J. Optim. Theory Appl., vol. 117, 2003, pp. 69-92.

[7] J. P. Lasalle, The Time Optimal Control Problem, Contributions to the Theory of Nonlinear Oscillations, Princeton University Press, Princeton, 1960, 1-24.

[8] X. Li and J. Yong, Optimal Control Theory for Infinite Dimensional Systems, Birkhauser, Boston, 1995, pp. 127-135.

[9] P. Lin and G. Wang, "Blowup time optimal control for ordinary differential equations", SIAM J. Control Optim., vol. 49, 2011, pp. 73-105.

[10] E. Meier and A. E. Bryson, Efficient algorithms for time-optimal rigid spacecraft reorientation problem, Journal of Guidance, Control, and Dynamics, vol. 13, 1990, pp. 859-866.
[11] T. Li and B. Rao. "A note on the exact synchronization by groups for a coupled system of wave equations", Mathematical Methods in the Applied Sciences, vol. 38, 2015, pp. 241-246.

[12] L. Hu, F. Ji and K. Wang. "Exact boundary controllability and exact boundary observability for a coupled system of quasilinear wave equations", Chinese Annals of Mathematics, vol.34(4), 2013, pp. 479-490.

[13] B. Z. Guo, D. H. Yang and L. Zhang. "On optimal location of diffusion and related optimal control for null controllable heat equation", Journal of Mathematical Analysis and Applications, vol. 433(2), 2016, pp. 1333-1349.

[14] M. Chen. "Bang bang property for time optimal control of the korteweg de vries burgers equation", appl.math. opt., vol. 2, 2016, pp. 1-16.

[15] L. Wang and Q. Yan. "Time Optimal Controls of Semilinear Heat Equation with Switching Control", Journal of Theory and Applications, vol. 165(1), 2015: 263-278.

[16] L. Wang and Q. Yan. "Bang-bang property of time optimal controls for some semilinear heat equation", Mathematics, 2015, 31(3): 477-499 